\title{
GIFTS EDU Ground-based Measurement Experiment
}

\author{
D. K. Zhou*, W. L. Smith, Sr. , L. J. Zollinger ${ }^{\dagger}$, R. J. Huppi ${ }^{\S}$, R. A. Reisse ${ }^{*}$, A. M. Larar ${ }^{*}$ X. Liu*, \\ J. J. Tansock, Jr. ${ }^{\dagger}$, S. M. Jensen ${ }^{\dagger}$, H. E. Revercomb ${ }^{\ddagger}$, W. F. Feltz ${ }^{\ddagger}$ and G. E. Bingham ${ }^{\dagger}$ \\ "NASA Langley Research Center, Hampton,VA 23681, USA (daniel.k.zhou@nasa.gov) \\ University of Wisconsin-Madison, WI 53706, USA \\ †Space Dynamics Lab., Utah State University, Logan, UT 84322, USA \\ ${ }^{\S}$ ZelTech, Inc., Hampton, VA 23681, USA
}

\begin{abstract}
Geosynchronous Imaging Fourier Transform Spectrometer (GIFTS) Engineering Demonstration Unit (EDU) is an imaging infrared spectrometer designed for atmospheric soundings. The EDU groundbased measurement experiment was held in Logan, Utah during September 2006 to demonstrate its extensive capabilities for geosynchronous and other applications.
\end{abstract}

OCIS codes: (280.0280) Remote sensing; (010.1280) Atmospheric composition

\section{Introduction}

Severe weather (e.g., hurricanes, tornadoes, floods, ice storms, etc.) impacts our daily lives. An accurate weather forecast is needed now more than ever for planning daily activities and for optimizing the preparation for the onset of lifethreatening and property-damaging weather (e.g., a hurricane landfall). The improvement of weather prediction requires high resolution temporal and spatial measurements of temperature, moisture, and wind profiles. High density and timely thermodynamic profile measurements are needed to forecast where and when severe convective storms will form, before they are visible on radar or in satellite cloud imagery. Quasi-continuous measurements of the moisture flux are needed for timely forecasts of storm intensity changes. Atmospheric wind profiles are needed to predict the storm's trajectory, and in the case of tropical storms and hurricanes, their landfall location and time. Based on these considerations, the Geosynchronous Imaging Fourier Transform Spectrometer (GIFTS) measurement concept [Smith et al., 2001] has been developed within the reach of new technologies for improving weather prediction accuracy.

Current and future planned satellite sensors, such as the Atmospheric InfraRed Sounder (AIRS) on the AQUA (2002), the Interferometer Atmospheric Sounding Instrument (IASI) on the METOP (2006), and the Cross-track Infrared Sounder (CrIS) on the NPP (2008) and NPOESS (2012) satellites, are attempting to fulfill the observation needs for accurate weather prediction. Valuable data obtained from the AIRS measurements have already shown the benefits of hyperspectral data. The GIFTS concept takes advantage of the geostationary satellite, an imaging Fourier transform spectrometer with large S/MWIR and LWIR focal plane detector arrays, and a near-infrared, band-filtered camera to revolutionize the spatial, spectral, and temporal observation of atmospheric conditions [Smith et al., 2005]. The GIFTS Engineering Demonstration Unit (EDU) has undergone a series of thermal vacuum tests followed by an extensive calibration phase. The success of the GIFTS EDU is not only critical to the GIFTS program itself but also for a demonstration of the new technologies incorporated in the EDU. NASA and NOAA are funding the completion and calibration of the GIFTS EDU as an important risk reduction effort for the Hyperspectral Environmental Suite (HES) program.

After all thermal vacuum tests and calibration experiments were completed in the summer 2006, a ground-based GIFTS EDU measurement experiment was conducted during September 2006. The GIFTS EDU team of scientists and engineers has written a white paper addressing the importance and significance of the experiment. A detailed operational plan was written (available from the GIFTS Program Office) and followed to collect the real atmospheric, lunar, and mountain-sky data needed to characterize and demonstrate the sensor system. This experiment is critical in demonstrating

the capabilities of the instrument, its calibration, and its associated data processing procedures including the retrieval of scientific products. An overview of this experiment is presented with some limited preliminary analyses to show that a successful experiment has been achieved.

\section{GIFTS EDU Ground-based Measurement Experiment}

The primary objectives of this experiment are to assess instrumental performance, to produce measured spectral radiance data utilizing the GIFTS EDU, to retrieve atmospheric conditions from the radiance data, and to validate the GIFTS EDU data products at all stages with ancillary data collected coincidently. Despite these primary goals, an important secondary objective is to demonstrate the capability of monitoring atmospheric spatial and temporal variations, which is the primary GIFTS measurement concept. In addition, a series of events was planned to characterize the sensor system such as the presence and levels of vibration and optical jitter and their affect on system performance and data products. In order to provide absolute calibration cross checks for validation of the GIFTS EDU measurements, the University of Wisconsin's 
AERIbago was brought to the Space Dynamics Laboratory (SDL) to obtain comparative measurements of the downwelling atmospheric radiance measurements with Atmospheric Emitted Radiance Interferometers (AERI) that have proven track records for the application. Details of the complete AERIbago measurement capabilities can be found on the web http://cimss.ssec.wisc.edu/AERIbago. It is a mobile laboratory containing a suite of meteorological instrumentation including an AERI, a Global Positioning System (GPS), a Vaisala GPS capable RS-92 rawinsonde launch capability, a Vaisala surface meteorological station, and a Vaisala ceilometer. There were two AERI instruments operated simultaneously during the GIFTS EDU ground-based measurement experiment: (1) one aligned with the GIFTS EDU field of view to less than approximate 3 degrees constant offset to view the sky using the same external pointing mirror used by the GIFTS EDU, and (2) one which does not use this external pointing mirror to directly view skyward toward the zenith direction as viewed by the GIFTS EDU via the external scene mirror. The AERI has about the same field of view as the field of regard of the GIFTS EDU and was used to account for the effects of the thermal vacuum chamber window and external scene pointing mirror on the absolute radiometric calibration of the GIFTS EDU. Figure 1 illustrates the setup of the GIFTS EDU chamber, AERIbago, AERI-05, and the external scene mirror.

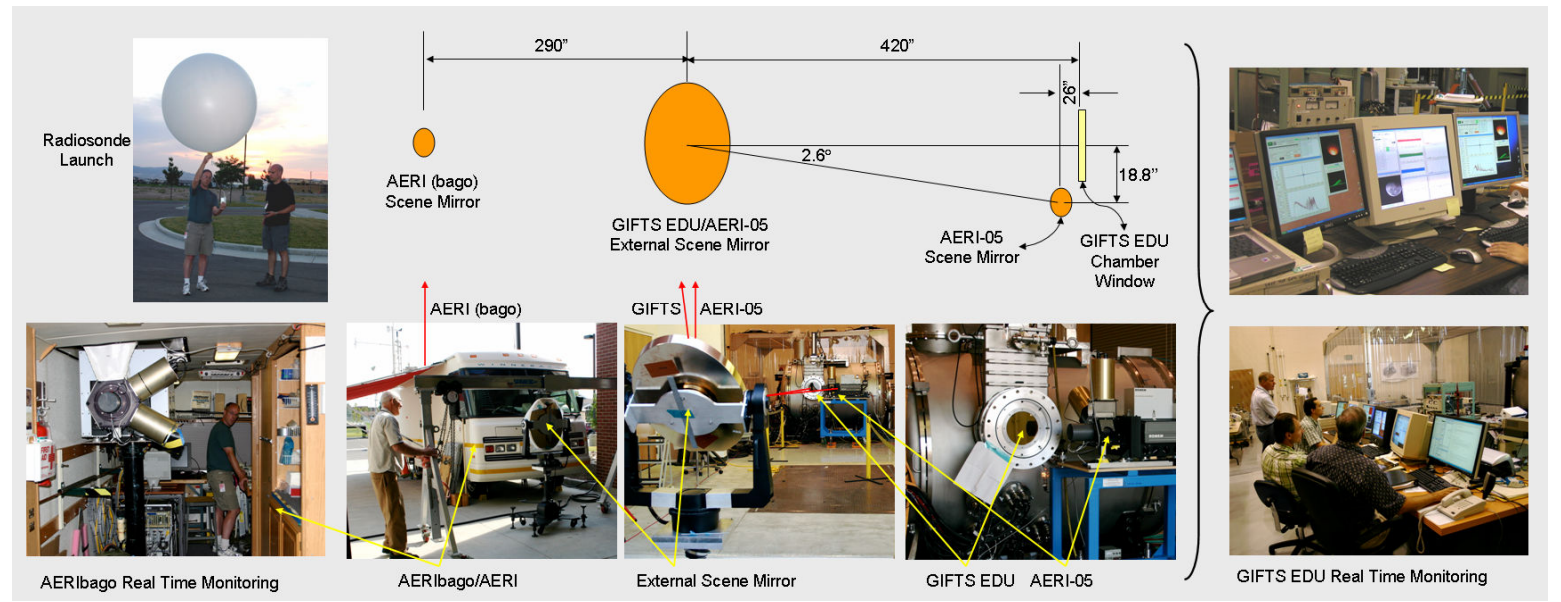

Fig. 1. GIFTS EDU ground-based measurement experiment setup (not to scale) to illustrate that GIFTS EDU, AERI-05, and AERI (bago) are lined up as close as possible to measure the same atmospheric conditions. Real time monitoring is to ensure the success of the experiment.

Prior to the science data collection, the GIFTS EDU is checked out and is made ready to collect quantified science data. Since this is a ground-based experiment, a Zinc Selenide ( $\mathrm{ZnSe}$ ) window is used to keep the GIFTS EDU under a space-simulated thermal vacuum condition in a chamber. This window is not needed when GIFTS is in space. Calibration data of the FTS sensor viewing through the ZnSe window were collected and analyzed prior to the science data collection events; these data are to be used to understand the window effects on the sensor performance relevant to the science data collected later on. A very important part of the preparation was to obtain the absolute spectral radiance responsivity for each detector pixel for application to measured atmospheric spatial scenes. This was accomplished by interfacing and sealing the precision High Accuracy Extended Source (HAES), extended black body calibration sources, to the window and performing measurements at several hot temperatures and a cold temperature to allow for comparison of HAES data to GIFTS EDU internal calibration source data. The comparison provides a basis for deriving spectral responsivity corrections for the internal source calibrations to account for optical path component differences between internal and external calibration sources. AERIbago facilities (including the radiosonde launch system) were checked out after arrival at the measurement site in Logan, Utah. AERI stand-alone measurements (under thin cirrus cloudy conditions) coordinated with a radiosonde launch were conducted to verify the readiness of the AERI (bago), the AERI-05, and the radiosonde launch systems. Both AERI radiances were compared with radiosonde-simulated radiances.

A series of 8 science and engineering data collection events was carefully planned to achieve the previously defined objectives of this experiment. The events included a Cross-Check Event (CCE), a Moon Viewing Event (MVE), a Moon Tracking Event (MTE), an Atmospheric Variation Event (AVE), an Outgas Monitoring Event (OME), a Moon-tracking and Sky-viewing Event (MSE), a Vibration Test Event (VTE), and a Jitter Test Event (JTE). Each event has its own objectives that are briefly described in Table 1 with associated performing dates. All these planned events were successfully performed. Quick look displays for the GIFTS EDU and both AERI instruments provided on-site scientists and engineers real time feed back to ensure the success of the experiment. Figure 2 shows some preliminary results obtained from the field measurements. Excellent agreement between the spectral data from GIFTS EDU and the AERI-05 sensors is clearly shown with a good temperature retrieval obtained from GIFTS EDU (courtesy of J. Taylor and B. Howell, University of Wisconsin). Extensive and intensive data analyses are ongoing to improve our understanding of GIFTS EDU, which is a great risk reduction effort for a future flight-GIFTS and any other instrument similar to the EDU. 
Table 1. GIFTS EDU ground-base experiment events, major objectives, and event performing dates.

\begin{tabular}{|c|c|c|}
\hline Event & Major Objectives & Date Performed \\
\hline $\mathrm{CCE}$ & $\begin{array}{l}\text { To compare the two sets of AERI measurements permitting a characterization of the spectral reflectance properties of } \\
\text { the scene mirror. The external scene mirror aligned AERI-05 and GIFTS EDU measurements provide comparative } \\
\text { data that are used to assess the spectral transmittance properties of the GIFTS EDU thermal vacuum chamber window } \\
(\mathrm{ZnSe}) \text { and optical path characteristics between the window and the GIFTS EDU external scene mirror. }\end{array}$ & $\begin{array}{r}5 \text { September } 2006 \\
6 \text { September } 2006 \\
25 \text { September } 2006\end{array}$ \\
\hline MVE & $\begin{array}{l}\text { To obtain high quality images of the Moon throughout GIFTS EDU spectral channels while the Moon moves across the } \\
\text { GIFTS EDU field of regard with a spectral resolution of } 9.2 \mathrm{~cm}^{-1} \text {, and to demonstrate GIFTS imaging capability. }\end{array}$ & 12 September 2006 \\
\hline MTE & $\begin{array}{l}\text { To obtain high quality images of the Moon throughout GIFTS EDU spectral channels (with a high spectral resolution } \\
\text { of } 0.57 \mathrm{~cm}^{-1} \text { ) while tracking the Moon with the GIFTS EDU field of regard, and to demonstrate GIFTS imaging } \\
\text { capability. }\end{array}$ & 11 September 2006 \\
\hline AVE & $\begin{array}{l}\text { To capture atmospheric temporal variation during dawn and/or dusk to monitor atmospheric diurnal variation when the } \\
\text { atmospheric temperature gradient is often most obvious. The sensor performance (e.g., noise level) and capability (e.g., } \\
\text { spectral coverage and resolution) are revealed through the temperature and moisture retrievals. }\end{array}$ & 13 September 2006 \\
\hline OME & $\begin{array}{l}\text { To monitor both temporal and spatial variations of the atmosphere with the GIFTS EDU when Sulfur Hexafluoride } \\
\left(\mathrm{SF}_{6}\right) \text { gas is released in the near field. }\end{array}$ & 18 September 2006 \\
\hline MSE & $\begin{array}{l}\text { To collect sky and moon data in the variable integration, variable frame mode of operation (i.e., Laser reference } \\
\text { controlled, OPD position and interval sampling), to understand how well simple integration time/offset normalization } \\
\text { algorithms work for two different scenes with different spectral content and different levels in the dynamic range for } \\
\text { reducing sampling error/noise due to vibration induced velocity variations of the interferometer scanning mirror. }\end{array}$ & 13 September 2006 \\
\hline VTE & $\begin{array}{l}\text { To determine the differences in the spectral data and noise characteristics with and without known vibration sources } \\
\text { and demonstrate that excellent performance of the interferometer is achieved under typical, but limited, vibration } \\
\text { conditions of known levels. }\end{array}$ & $\begin{array}{l}18 \text { September } 2006 \\
25 \text { September } 2006\end{array}$ \\
\hline JTE & $\begin{array}{l}\text { To determine the optical and pointing jitter characteristics of the sensor and to demonstrate imaging capability. The } \\
\text { objectives are to determine effects of discrete frequency jitters on the spectra and the overall affects of jitter in the } \\
\text { spatial image at sharp edges in a stable scene. }\end{array}$ & $\begin{array}{l}28 \text { September } 2006 \\
29 \text { September } 2006\end{array}$ \\
\hline
\end{tabular}
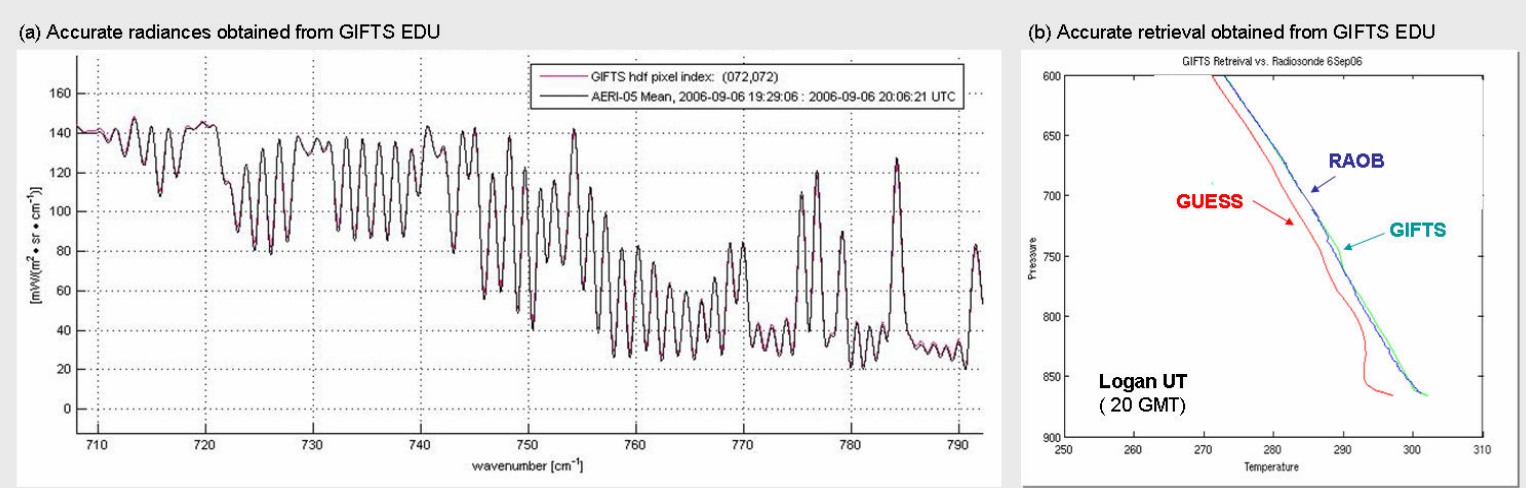

Fig. 2. Panel (a) plots GIFTS EDU and AERI-05 radiance comparison and panel (b) plots GIFTS EDU temperature retrieval compared with a coincident radiosonde observation from the Cross Check Event (CCE), 6 September 2006.

\section{Conclusion}

The GIFTS EDU ground-based measurement experiment was carefully planned and successfully conducted. The GIFTS proof of concept is being successfully demonstrated with collection of data from the ground-based measurements and the thermal-vacuum performance and calibration tests. Extensive and detailed data analyses are ongoing. Full analysis of the collected data will provide the necessary information to refine the design of the sensor system and allow construction of a high-performance, low-risk flight instrument in the near future.

Acknowledgments: This experiment is supported by the NASA Headquarters and NASA Langley Research Center. The authors express their sincere thanks to the GIFTS team members from various organizations. The personnel who contributed to this study are too numerous to mention by name, nonetheless their personal contributions are greatly appreciated.

\section{Reference:}

Smith, W. L.; F. W. Harrison, H. E. Revercomb, and G. E. Bingham (2001), Geostationary Imaging Fourier Transform Spectrometer (GIFTS) - The New Millennium Earth Observing-3 Mission, Proc. of IRS 2000: Current Problems in Atmospheric Radiation, A Deepak Publishing, Hampton, Virginia, 81-84.

Smith, W. L. Sr., H. E. Revercomb, D. K. Zhou, H. L. Huang (2005), Hyperspectral sounding - a revolutionary advance in atmospheric remote sensing, Proc. SPIE 5655, 1-11. 